\title{
Materializing Rival Ground States in the Barlowite Family of Kagome Magnets
}

\author{
R Smaha ${ }^{1}$, W He ${ }^{1}$, J Jiang ${ }^{1}, C_{\text {Titus }}{ }^{1}$, J Wen ${ }^{1}$, Yee $^{1}$ \\ ${ }^{1}$ Stanford University, Stanford, CA \\ rsmaha@stanford.edu
}

Quantum magnets display exotic phases that may be strongly influenced by small differences in structure and composition. The quantum spin liquid (QSL) is an unusual magnetic ground state, characterized by long-range quantum entanglement of the spins but a lack of long-range magnetic order down to $T=0 \mathrm{~K}$.[1] Materials with a kagome arrangement of $\mathrm{Cu} 2+$ ions are prime candidates as the QSL ground state is believed to be attainable in highly frustrated spin-1/2 systems. Newly synthesized crystalline barlowite $(\mathrm{Cu} 4(\mathrm{OH}) 6 \mathrm{FBr})$ and $\mathrm{Zn}$-substituted barlowite demonstrate the delicate interplay between singlet states and spin order on the spin-1/2 kagome lattice.[2] We find that when the bonds of the kagome lattice are modulated with a periodic pattern, new quantum ground states emerge. Comprehensive structural and magnetic measurements (including single crystal and powder X-ray diffraction, powder neutron diffraction, and single crystal neutron scattering) address open questions about their crystal and magnetic structures. We reveal a clear structure-properties relationship between two variants of barlowite with distinct low-temperature structures (orthorhombic Pnma and hexagonal P63/m).[3] This hexagonal variant is novel, and we are able to grow large single crystals. Its phase transition involves a subtle symmetry lowering, and its kagome lattice contains a motif of distorted and undistorted triangles, for which numerical simulations predict a pinwheel valence bond crystal state instead of a QSL state. The presence of interlayer spins eventually leads to novel pinwheel $\mathrm{q}=0$ magnetic order, which we elucidate with single crystal neutron scattering. Sizable single crystals of QSL candidate $\mathrm{Zn}$-substituted barlowite $(\mathrm{Cu} 3.44 \mathrm{Zn} 0.56(\mathrm{OH}) 6 \mathrm{FBr})$ were grown for the first time and have an intermediate level of $\mathrm{Zn}$ substitution compared to $\mathrm{Cu} 3.05 \mathrm{Zn} 0.95(\mathrm{OH}) 6 \mathrm{FBr}$ and herbertsmithite. Site-specific X-ray anomalous dispersion, extended X-ray absorption fine structure, and X-ray absorption spectroscopy measurements demonstrate that $\mathrm{Zn} 2+$ occupies a perfect trigonal prismatic interlayer site with minimal amount of antisite defects. They also demonstrate that the interlayer $\mathrm{Zn} 2+$ coordination in $\mathrm{Zn}$ substituted barlowite is distinct from that in herbertsmithite. Significantly, no structural transition is observed at low temperature in either composition of $\mathrm{Zn}$-substituted barlowite, and no magnetic transition occurs down to $\mathrm{T}=$ $0.1 \mathrm{~K}$, indicating a surprising robustness of the QSL against interlayer $\mathrm{Cu} 2+$ impurities. These samples span a spectrum of quantum spin liquidity, allowing this exotic ground state to be probed systematically. References: [1] Norman, M. R. (2016). Rev. Mod. Phys. 88, 041002. [2] Smaha, R. W. et al. (2018). J. Solid State Chem. 268, 123129. [3] Smaha, R. W. et al. (2020). npj Quantum Materials 5, 23.

Acta Cryst. (2020). A76, a168 